\title{
Leptin Affects Intestinal Epithelial Cell Turnover in Correlation With Leptin Receptor Expression Along the Villus-Crypt Axis After Massive Small Bowel Resection in a Rat
}

\author{
IGOR SUKHOTNIK, ARNOLD G. CORAN, JORGE G. MOGILNER, BENHOOR SHAMIAN, RAHEL KARRY, MICHAEL LIEBER,
} AND RON SHAOUL

\begin{abstract}
Departments of Pediatric Surgery [I.S., J.G.M.] and Pediatrics [R.S.], Bnai Zion Medical Center, Haifa 31048, Israel; Laboratory of Intestinal Adaptation and Recovery [I.S., B.S., R.K., M.L.], Technion-Israel Institute of Technology, The Ruth \& Bruce Rappaport Faculty of Medicine, Haifa 31096, Israel; Section of Pediatric Surgery [A.G.C.], University of Michigan Medical School, Ann Arbor, Michigan 48109
\end{abstract}

\begin{abstract}
In this study, we examine the responsiveness of intestinal epithelial cell turnover to leptin (LEP) in correlation with leptin receptor (LEPr) expression along the villus-crypt axis in a rat with short bowel syndrome (SBS). Adult rats underwent either a 75\% intestinal resection or a transection. SBS-LEP rats underwent bowel resection and were treated with LEP starting from the fourth postoperative day. Parameters of intestinal adaptation, enterocyte proliferation, and enterocyte apoptosis were determined at sacrifice. RTPCR technique was used to determine Bax and Bcl-2 gene expression in ileal mucosa. Villus tips, lateral villi, and crypts were separated using laser capture microdissection. LEPr expression for each compartment was assessed by quantitative real-time PCR (Taqman). Treatment with LEP significantly stimulated all parameters of adaptation. LEPr expression in crypts significantly increased in SBS rats (vs Sham rats) and was accompanied by a significant increase in enterocyte proliferation and decreased apoptosis after LEP administration. A significant increase in LEPr expression at the tip of the villus in SBS rats was accompanied by decreased cell apoptosis. In conclusion LEP accelerated enterocyte turnover and stimulated intestinal adaptation. The effect of LEP on enterocyte proliferation and enterocyte apoptosis correlated with receptor expression along the villus-crypt axis. (Pediatr Res 66: 648-653, 2009)
\end{abstract}

$\mathrm{E}$ xtensive studies in various experimental models of short bowel syndrome (SBS) have established that enterocyte apoptosis plays a crucial role during the process of postresection intestinal adaptation $(1,2)$. Despite the need for intestinal hyperplasia, the mitotic stimuli fail to suppress programmed cell death (3). Many investigators have described increased enterocyte apoptosis as a mechanism that counterbalances the increased enterocyte proliferation to reach a new homeostatic set during intestinal adaptation (4). Increased apoptosis promotes disposal of genetically aberrant stem cells and prevents tumorogenesis $(3,4)$.

Under normal circumstances, renewal of the small intestinal epithelium takes place continuously in anatomically distinct crypt-villus units. Proliferation is restricted to mucosal invaginations known as crypts of Lieberkühn. All epithelial cells in

Received May 4, 2009; accepted August 25, 2009.

Correspondence: Igor Sukhotnik, M.D., Department Pediatric Surgery, Bnai Zion Medical Center, 47 Golomb St., P.O.B. 4940, Haifa 31048, Israel; e-mail: igor-dr@ internet-zahav.net

Supported by an Israel Science Foundation (ISF) and Albert Goodstein research grants. each crypt are derived from an uncertain number of multipotential stem cells located at or near the base of the crypt (5). Stem cells in the small intestinal epithelium are known to differentiate into columnar, mucous, enteroendocrine, and Paneth cells as they move from a crypt up the adjacent villus (6). It takes 2 to $5 \mathrm{~d}$ for members of these lineages to travel from the crypt to the apex of the villus (7) where they are removed by apoptosis and/or exfoliation. Apoptosis probably accounts for the bulk of cell loss in the gut and is a central feature of the regulation of cell number in the adult gastrointestinal tract (8). After exposure of cells to stimuli that trigger programmed cell death, cytochrome $c$ is rapidly released from mitochondria into the cytoplasm, activating proteolytic molecules known as caspases, which are crucial for the execution of apoptosis. Augmented expression of Bcl-2 or the related protein bcl- $\mathrm{x}_{\mathrm{L}}$ act in situ on mitochondria to prevent the release of cytochrome $c$ and thus caspase activation (9), whereas Bax localizes to mitochondria and induces the release of cytochrome $c$, activation of caspase-3, membrane blebbing, nuclear fragmentation, and eventually cell death (10).

Intestinal adaptation after bowel resection results in increased proliferation of intestinal epithelial cells, as well as enhanced programmed cell death, suggesting accelerated cell turnover. The result of these actions helps to reshape the crypt-villus structures to more elongated and functionally active units. The regulation of the balance between cell production and cell loss through apoptosis after bowel resection is complex and the precise factors that guide this adaptive process remain unclear $(11,12)$. In particular, it is not clear where many of these trophic factors act along the crypt-villus axis. Recent evidence suggests that several growth factors such as keratinocyte growth factor (13) and epidermal growth factor (14) are differentially expressed along the crypt-villus axis. This suggests that different trophic factors may act on different locations along this axis. We have shown recently that TGF-alpha (TGF- $\alpha$ ) simulates enterocyte turnover in accordance to epidermal-growth factor receptor expression along the villus-crypt axis (15).

Abbreviations: LEP, leptin, SBS, short bowel syndrome 
The obese gene protein product leptin (LEP) is secreted from adipocytes and acts primarily on the hypothalamus regulating energy expenditure, food intake, and body weight (16). Although the intestine is not a classic target tissue for LEP, extensive studies in various experimental models have established that LEP determines important physiologic effects on intestinal growth, cell maturation, and differentiation $(17,18)$. In our previous work, we have shown that LEP stimulates intestinal adaptation after massive small bowel resection in a rat (19). However, the mechanisms of this positive effect remain poorly understood.

The purpose of this study was to evaluate the effects of LEP on enterocyte turnover (proliferation and apoptosis) in conjunction with LEP receptor (LEPr) expression along the villus-crypt axis after massive small bowel resection in the rat.

\section{MATERIALS AND METHODS}

Animals. Rappaport Faculty of Medicine (Technion, Haifa, Israel) Institutional Animal Care and Use Committee approved the animal facilities and protocols. Adult Sprague-Dawley male rats weighing 240 to $260 \mathrm{~g}$ were kept in individual stainless steel cages at constant temperature and humidity, and a 12-h light-dark cycle was maintained. Rats were put on fasts $12 \mathrm{~h}$ before the experiment with free access to water. General anesthesia was induced with ketamine (i.p. $90 \mathrm{mg} / \mathrm{kg}$ ) and xylasine (IP $15 \mathrm{mg} / \mathrm{kg}$ ).

Experimental design. Forty rats were randomly assigned to one of three groups: group A, Sham rats underwent bowel transection (Sham, $n=14$ ); group B, SBS animals underwent bowel resection (SBS, $n=13$ ); and group C, SBS-LEP rats underwent bowel resection (SBS-LEP, $n=13$ ) and were treated with LEP given i.p. at a dose of $50 \mathrm{mg} / \mathrm{kg}$ from $\mathrm{d} 4$ to $\mathrm{d} 14$.

Surgical procedure. Rats underwent one of two surgical procedures: bowel transection followed by reanastomosis or $75 \%$ bowel resection. Using sterile techniques, the abdomen was opened using a midline incision. In Sham rats, the mid-small bowel was transected and reanastomosed without bowel resection. In SBS animals, a 75\% mid-small bowel resection was performed similar to that previously described. This consisted of a resection of the bowel between $5 \mathrm{~cm}$ distal to the ligament of Treitz and $10 \mathrm{~cm}$ proximal to the ileocecal valve. Bowel continuity was restored by end-to-end anastomosis using 6-0 absorbable suture (Vicryl, Ethicon Corporation, USA). For all operations, the abdominal cavity was closed in two layers with a running suture of $3 / 0$ Vicryl (Ethicon Corporation, USA). Postoperative rats were allowed ad libitum water and a liquid diet. The rats were killed on $\mathrm{d} 15$ by i.p. injection of pentobarbital $(75 \mathrm{mg} / \mathrm{kg})$.

Intestinal adaptation parameters. The small bowel was rapidly removed, rinsed with cold isotonic saline, and divided into two segments: jejunum proximal to anastomosis and terminal ileum. The intestine was split on the antimesenteric border, washed with cold saline, dried, and each segment was weighed. The mucosa was scraped from the underlying tissue using a spatula (Sigma Chemical Co., Israel). Mucosal samples were homogenized with TRIzol reagent. DNA and protein were extracted by Chomczynski method (20) and were expressed as micrograms per centimeter of bowel per $100 \mathrm{~g}$ of body weight.

Histologic examination. Histologic sections were prepared from the proximal jejunum, distal ileum, and comparable sites in the control animals. Segments of small bowel were fixed for $24 \mathrm{~h}$ in $10 \%$ formalin and processed into standard paraffin blocks. Five-micron tissue slices were stained with hematoxylin and eosin. The villus height and crypt depth were measured using Image Pro Plus 4 image analysis software (Media Cybernetics, Baltimore, MD). Ten villi and crypts in each section were measured, and the mean reading was recorded in microns.

Laser-capture microdissection and RNA preparation. Fifteen-micronthick sections were mounted onto special RNase-free and UV-treated membrane-covered slides (PALM Technologies, Bernried, Germany) and immediately fixed in ice-cold $70 \%$ ethanol for $2 \mathrm{~min}$. After incubation for $60 \mathrm{~s}$ in $1 \%$ cresyl violet acetate, the sections were then dehydrated in an ethanol series (70 and $100 \%$ on ice) and left to air-dry briefly. Slides were stored at $-80^{\circ} \mathrm{C}$ until microdissection. Sections were laser dissected within $3 \mathrm{~d}$. Slides were observed using a Zeiss Axiovert $200 \mathrm{M}$ inverted laser-capture microscope and visualized on a monitor using the PALM Robo-Software. Villus tips, lateral villi, and crypts were separated using the laser. RNA was extracted using the RNeasy Microkit (Qiagen) and microdissection protocol. All samples were put at $-80^{\circ} \mathrm{C}$ for long-term storage.

$\boldsymbol{R T}$-PCR. The quality of RNA was evaluated using the Experion automated electrophoresis system (BioRad). Five micrograms RNA were reverse transcribed to cDNA at $37^{\circ} \mathrm{C}$ using $200 \mu \mathrm{M}$ deoxynucleotides (Sigma Chemical Co., St. Louis, MO), $5 \mu \mathrm{M}$ random hexamers (Amersham Pharmacia Biotech, Piscataway, NJ), 20 U RNAguard (Amersham Pharmacia Biotech), and $200 \mathrm{U} / \mu \mathrm{L}$ Moloney murine leukemia virus-reverse transcriptase (US Biochemicals, Cleveland, $\mathrm{OH})$. Thermal cycler settings were optimized to ensure products were in the linear phase of production.

Real-time PCR. Expression of long form of the LEPr levels was determined by quantitative real-time PCR on the cDNA samples using TaqMan assay-on demand kit (ABsolute Blue QPCR ROX Mix (ROX Dye) from ABgene, Epsom, UK) with the ABI-PRISM 7000 (Applied Biosystems, Foster City, CA). Single-exon primers (from PrimerDesing Ltd, UK) with distance from 3'UTR-1064 bp (sense primer, GCAGGGCTGTATGTCATTGTA; anti-sense primer, GAACATGGTCCCAAAACAACTT) were designed to produce an amplicon 109 bp. 18S (5'AGGAATTGACGGAAGGGCAC, 3'GTGCAGCCCCGGACATCTAAG) was used to access equal cDNA loading for each compartment. The primers are specific for the long form of the LEPr and they do not reside in the same exon; otherwise, amplification from contaminating DNA cannot be distinguished from amplification of cDNA.

Crypt cell proliferation and enterocyte apoptosis. Rats were injected with standard 5-bromodeoxyuridine (5-BrdU) labeling reagent (Zymed Lab, Inc, $\mathrm{CA})$ at a dose of $1 \mathrm{~mL}$ per $100 \mathrm{~g}$ body weight, $2 \mathrm{~h}$ before sacrifice. Tissue slices $(5 \mu \mathrm{m})$ were deparaffinized with xylene, rehydrated with graded alcohol, and stained with a biotinylated monoclonal anti-BrdU antibody system using BrdU Staining Kit (Zymed Lab, Inc, CA). An index of proliferation was determined as the ratio of crypt cells staining positively for BrdU per 10 crypts.

Additional 5- $\mu \mathrm{m}$ thick sections were prepared to establish the degree of enterocyte apoptosis. Immunohistochemistry for Caspase-3 (Caspase-3 cleaved concentrated polyclonal antibody; dilution 1:100; Biocare Medical, Walnut Creek, CA) was performed to identify apoptotic cells using a combination of streptovidin-biotin-peroxidase method according to manufacturers' protocols. Expression of epithelial cell apoptosis is expressed as the total number of apoptotic cells along this axis per 10 villi and 100 crypts. In some cases, a more detailed analysis of the location of apoptosis was performed using previously established techniques (21). For this, apoptosis along the villi were differentiated between the lower one-third of the villi (lateral villi) and upper one-third of the villi (villi tips). Apoptosis was recorded as the number of apoptotic cells per 10 villi. A qualified pathologist blinded as to the source of intestinal tissue performed all measurements.

Expression of Bax and Bcl-2 genes. Total RNA was isolated from frozen mucosal samples (proximal jejunum and distal ileum) using TRIzol reagent (GIBCO BRL, USA), as described by Chomczynski (20). A portion of total RNA $(2 \mu \mathrm{g}$ in a total volume of $25 \mu \mathrm{L})$ was reverse transcribed using Moloney murine leukemia virus (MMLV) First strand cDNA Synthesis Kit (Gene Choice, Inc. Frederick, MD). After PCR, the amplified product $(5 \mu \mathrm{L})$ was run on a $2 \%$ agarose gel stained with ethidium bromide and photographed. The level of Bax and Bcl-2 gene expression was expressed as the ratio of the gray density of the objective gene over the gray density of $18 \mathrm{~S}$ at densitometry. The sequences for the specific genes were as follows: Bax $5^{\prime}$ ATGGACGGGTCCGGGGAGCA, 3'ATGGACGGGTCCGGGGAGCA, Bcl-2 5'TGAGGCCCTGTCTGCTTCTG, 3'AGGCTCCCGGGGCAGTCATGA (all primers were purchased from Sigma Chemical Co., Sigma Chemical Co.-Aldrich Biotechnology LP). Because 18S RNA is expressed at much higher levels than most mRNAs, the 18S rRNA primers was diluted 1:10 to bring the RT-PCR products within the same exponential range of amplification.

Statistical analysis. The data are expressed as the mean \pm SEM. A one-way ANOVA test followed by Bonferroni post hoc test was used for statistical analysis with $p$ value $<0.05$ considered statistically significant.

\section{RESULTS}

Parameters of intestinal adaptation. Massive small bowel resection resulted in a significant decrease in body weight. SBS rats (group B) had a significantly lower final body weight compared with Sham rats $(p<0.001$, Table 1$)$. Treatment with LEP resulted in a small but significant increase in final body weight compared with SBS rats $(p<0.05)$. SBS rats (group B) demonstrated a significant increase in overall bowel 
Table 1. Parameters of intestinal adaptation

\begin{tabular}{|c|c|c|c|}
\hline Parameters & Sham (group A, $n=14$ ) & $\mathrm{SBS}($ group B, $n=13)$ & SBS-LEP (group C, $n=13$ ) \\
\hline Body weight (\%preoperative) & $114 \pm 1$ & $102 \pm 2^{*}$ & $106 \pm 2 * \dagger$ \\
\hline \multicolumn{4}{|l|}{ Bowel weight $(\mathrm{mg} / \mathrm{cm} / 100 \mathrm{~g})$} \\
\hline Jejunum & $19 \pm 1$ & $106 \pm 5^{*}$ & $117 \pm 4^{* \dagger}$ \\
\hline Ileum & $23 \pm 2$ & $54 \pm 7 *$ & $57 \pm 4^{*}$ \\
\hline \multicolumn{4}{|l|}{ Mucosal weight $(\mathrm{mg} / \mathrm{cm} / 100 \mathrm{~g})$} \\
\hline Jejunum & $7.3 \pm 0.4$ & $36.5 \pm 2.5^{*}$ & $42.4 \pm 2.3^{* \dagger}$ \\
\hline Ileum & $8.18 \pm 0.5$ & $17.6 \pm 1.7^{*}$ & $21.8 \pm 0.8 * \dagger$ \\
\hline \multicolumn{4}{|l|}{ Mucosal DNA $(\mu \mathrm{g} / \mathrm{cm} / 100 \mathrm{~g})$} \\
\hline Jejunum & $85 \pm 15$ & $328 \pm 58^{*}$ & $361 \pm 29 *$ \\
\hline Ileum & $145 \pm 29$ & $184 \pm 20^{*}$ & $229 \pm 12 * \dagger$ \\
\hline \multicolumn{4}{|l|}{ Mucosal protein $(\mu \mathrm{g} / \mathrm{cm} / 100 \mathrm{~g})$} \\
\hline Jejunum & $825 \pm 85$ & $2883 \pm 204^{*}$ & $4376 \pm 1008 *$ \\
\hline Ileum & $739 \pm 148$ & $1478 \pm 147^{*}$ & $2244 \pm 347 * \dagger$ \\
\hline \multicolumn{4}{|l|}{ Villus height $(\mu \mathrm{m})$} \\
\hline Jejunum & $239 \pm 21$ & $340 \pm 18^{*}$ & $386 \pm 25 * \dagger$ \\
\hline Ileum & $178 \pm 18$ & $219 \pm 12^{*}$ & $295 \pm 32 * \dagger$ \\
\hline \multicolumn{4}{|l|}{ Crypt depth $(\mu \mathrm{m})$} \\
\hline Jejunum & $89 \pm 7$ & $116 \pm 4^{*}$ & $119 \pm 7 *$ \\
\hline Ileum & $85 \pm 6$ & $98 \pm 8$ & $144 \pm 2 * \dagger$ \\
\hline
\end{tabular}

Values are mean \pm SEM.

$* p<0.05$ SBS $v s$ Sham rats.

$\dagger p<0.05$ SBS-LEP $v s$ SBS rats.

and mucosal weight in jejunum (5-fold increase; $p<0.001$ ) and in ileum (3-fold and 2-fold increase, respectively; $p<$ 0.001 ); mucosal DNA and protein in jejunum (4-fold and 3 -fold, respectively; $p<0.05$ ) and in ileum (27\% and 2-fold, respectively; $p<0.05$,), villus height in jejunum (42\%, $p<$ $0.05)$ and in ileum $(23 \%, p<0.05)$; and crypt depth in jejunum $(30 \%, p<0.05)$ compared with Sham rats (group A) (Table 1). Treatment with LEP resulted in additional bowel growth. SBS-LEP rats (group C) demonstrated an additional increase in overall bowel weight in jejunum $(10 \%, p<0.05)$ and mucosal weight in jejunum and ileum (16 and 24\%, $p<$ 0.05 and $p<0.001$, respectively), mucosal DNA and protein in ileum (10 and 52\%, $p<0.05$, respectively), villus height and crypt depth in ileum (35 and $16 \%$, respectively, $p<0.05$ ) compared with SBS-untreated animals (group B).

Enterocyte proliferation and apoptosis in jejunum. SBS rats (group B) demonstrated a significant increase in cell proliferation rate in jejunum $(22 \%, p<0.005)$ and concomitant increase in cell apoptosis $(62 \%, p<0.05)$ compared with Sham rats (group A). Treatment with LEP (group C) resulted in an additional increase in cell proliferation rate in jejunum $(31 \%, p<0.005)$ and in a significant decrease in cell apoptosis $(43 \%, p<0.05)$ compared with SBS rats (group B).

Long form of the LEPr expression and enterocyte turnover in ileal crypts. Bowel resection (group B) resulted in a significant increase in enterocyte proliferation $(16 \%, p<$ 0.005 ) and concomitant increase in cell apoptosis (4-fold increase, $p<0.05$ ) in ileum compared with Sham rats (group A). Adaptation of residual bowel in the resected group (group B) was manifested by a 3-fold increase in LEPr mRNA expression within crypt cell population ( $v s$ Sham rats) (Fig. 1). This increase of the long form of LEPr expression coincided with increased cell proliferation $(36 \%, p<0.05)$ and decreased cell apoptosis (3-fold decrease, $p<0.05$ ) after LEP administration (group C).
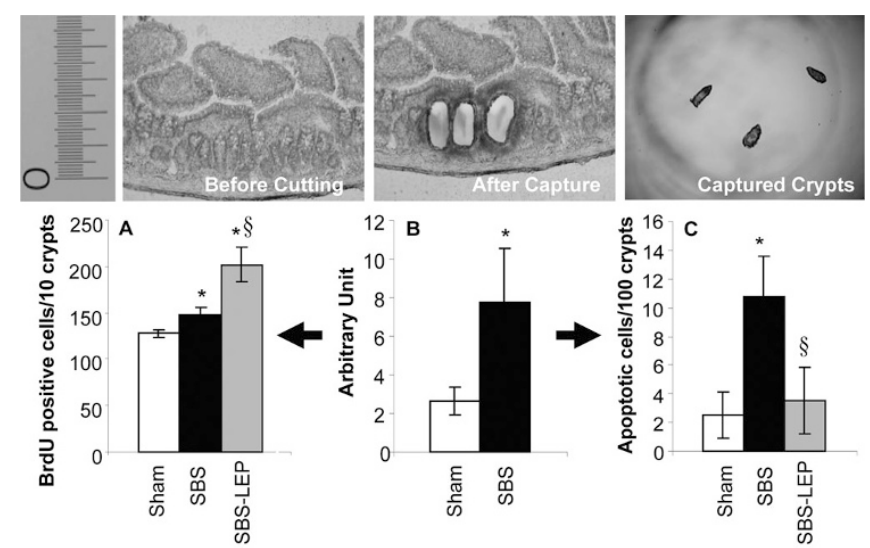

Figure 1. Relationship between leptin receptor mRNA expression $(B)$ and enterocyte proliferation $(A)$ and apoptosis $(C)$ in crypts after massive small bowel resection and treatment with leptin. Values are mean \pm SEM. Magnification 1:100. $* p<0.05$, SBS $v s$ Sham rats; $\$ p<0.05$, SBS-LEP $v s$ SBS rats.

Long form of the LEPr expression and enterocyte apoptosis in ileal villi. SBS rats demonstrated a significant increase in cell apoptotic rates in villus tips in the ileum $(65 \%, p<$ 0.05 ) compared with Sham rats (group A). Similar to the crypt compartment, LEPr expression was up-regulated in villus tips of SBS rats (group B) compared with Sham rats (Fig. 2). This increase in LEPr expression coincided with decreased cell apoptosis in villus tips $(39 \%, p<0.05)$ after LEP administration (group C). Cell apoptosis did not change significantly in lateral villi in SBS rats compared with Sham rats. In contrast to crypts and villus tips, long form of the LEPr expression remained unchanged in lateral villi in resected rats compared with Sham rats. In relation to LEPr expression, cell apoptosis remain unchanged in this compartment after LEP administration (Fig. 2).

Expression of apoptosis related genes. A significant increase in cell apoptosis in SBS rats (group B) compared with 

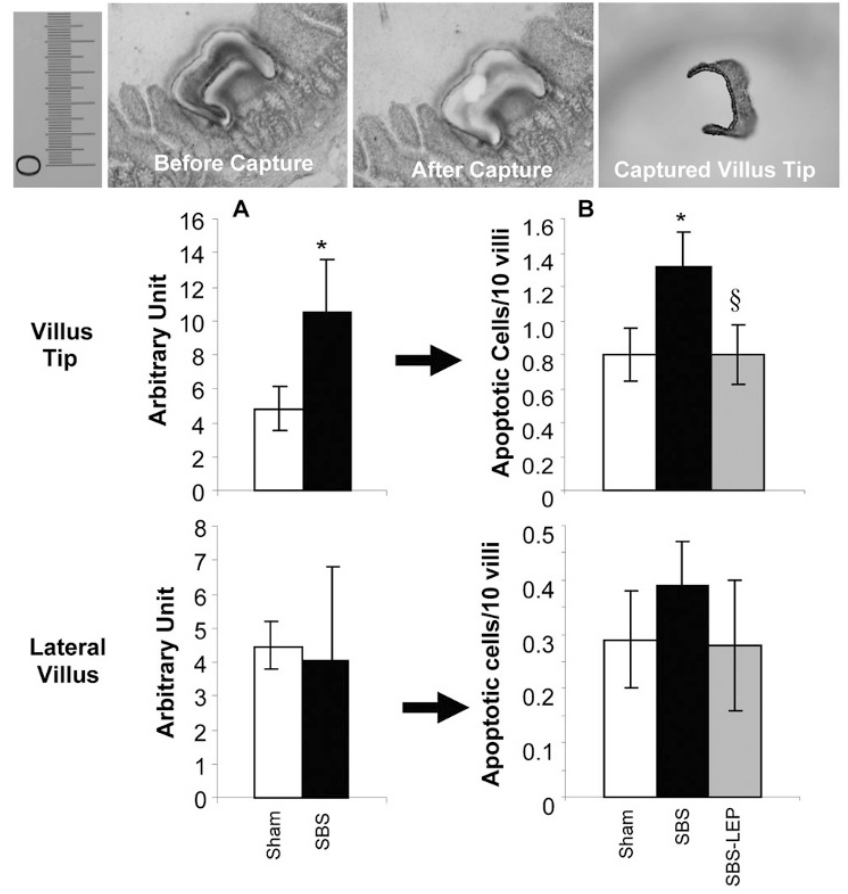

Figure 2. Effect of bowel resection and treatment with leptin on enterocyte apoptosis $(B)$ in correlation with leptin receptor expression $(A)$ in villus tips and in lateral villi. Values are mean \pm SEM. Magnification $1: 100 .{ }^{*} p<0.05$, SBS $v s$ Sham rats; $\$ p<0.05$, SBS-LEP $v s$ SBS rats.

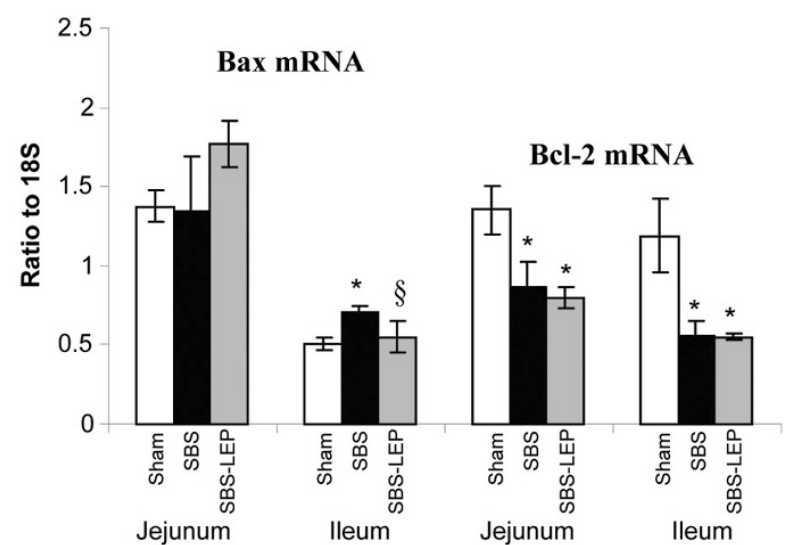

Figure 3. Effect of bowel resection and treatment with leptin on expression of Bax and Bcl-2 in ileal mucosal samples. Values are mean \pm SEM. $* p<$ 0.05 , SBS $v s$ Sham rats; $\$ p<0.05$ SBS- LEP $v s$ SBS rats.

Sham rats (group A) was accompanied by a significant increase in proapoptotic Bax gene expression in the ileum $(p<$ $0.05)$ and a concomitant decrease in Bcl-2 gene expression in jejunum $(36 \%, p<0.05)$ and the ileum (2-fold decrease, $p<$ 0.05) compared with Sham rats (group A) (Fig. 3). Treatment with LEP (group C) did not significantly change Bcl-2 gene expression but led to significant down-regulation in Bax mRNA expression in ileum compared with SBS animals $(p<$ $0.05)$.

\section{DISCUSSION}

Although intestinal transplantation has emerged as a feasible alternative in the treatment of children with SBS in the last two decades, the single most important factor contributing to individual outcome in these patients is the capacity of the intestinal remnants to undergo an adaptation process. Intestinal adaptation is defined as a process of progressive recovery from intestinal failure after bowel resection and includes morphologic and functional changes $(11,12)$ Morphologic changes include lengthening of the villi and deepening of the crypts, increased enterocyte proliferation, and increased migration of enterocytes along the villi. Functional adaptation results in the enhanced absorption of nutrients by isolated enterocytes (21-23).

LEP is a nutritionally regulated $16-\mathrm{kD}$ cytokine that is secreted from adipocytes and is found to highly correlate with body mass index in rodents, and in lean and obese humans (16). LEP regulates food intake and energy expenditure by providing afferent signals to the thalamus. LEP controls feeding and neuroendocrine function and regulates adiposity through activation of a long form receptor. In addition to adipose tissue, both the LEP and LEPr have also been found in many other tissues, which suggests possible important peripheral actions in rodent and humans (23). The fact that LEP is present in human milk, that gastrointestinal mucosa is capable of producing this growth factor (24), and that LEPrs are expressed in both basolateral and brush border membranes of the enterocytes (23) suggests its potential role in small intestinal growth and development. We have recently shown that parenteral LEP induces intestinal regrowth, stimulates cell proliferation, and inhibits enterocyte apoptosis in rat models of SBS (19). However, the mechanisms of this positive effect remain poorly understood. In particular, it is not clear where this factor acts along the crypt-villus axis and whether it acts on different locations along this axis in correlation with the LEPr, which may be differentially expressed along the axis.

The purpose of this study was to investigate the effects of LEP on intestinal mucosal homeostasis in conjunction with long form of the LEPr expression along the villus-crypt axis after bowel resection in rats. Cell proliferation and apoptosis were measured in crypts of the remnant ileum to characterize enterocyte turnover, and enterocyte apoptosis was determined in villus tips and lateral villi. Similar to our previous experiment $(15,19)$, this study showed that massive intestinal resection results in significant structural adaptation characterized by increased bowel and mucosal weights and increased mucosal DNA and protein. Increase in DNA and protein content in our model suggests that hyperplasia was the predominant adaptive response. Increased crypt depth in both jejunum and ileum suggests increased cell proliferation and was correlated with an increased enterocyte proliferation index. Administration of LEP significantly enhanced structural intestinal adaptation. The observed changes in weight loss, and particularly the lower degree of weight loss in the SBS-LEP group, may suggest an improvement in SBS adaptation in the LEP treated group, although specific absorption studies would need to be performed in future research to prove this. LEP-treated rats demonstrated an additional increase in bowel and mucosal weight, mucosal DNA and protein in jejunum. Increased villus height and crypt depth are the result of increased proliferation and accelerated migration along the villus and are a marker for 
an increased absorptive surface area. Like our previous reports $(15,19)$, apoptosis in remaining bowel segments increased significantly after massive small bowel resection in both jejunum and ileum compared with Sham rats. We have also shown a significant increase in proapoptotic Bax gene expression and down-regulation of antiapoptotic gene Bcl-2, which may be responsible for enhanced cell apoptosis. Together with increased enterocyte proliferation, enhanced programmed cell death suggests accelerated cell turnover and is considered by many investigators as a mechanism that counterbalances the increased enterocyte mass to reach a new homeostatic set, to promote disposal of genetically aberrant stem cells, and to prevent tumorogenesis $(3,4)$. Administration of the LEP to SBS rats reversed the increase in enterocyte apoptosis observed with SBS alone, bringing levels to that of Sham rats. Interestingly, this observed LEP-associated decline in cell apoptosis was predominately in the crypt region and was reflected morphologically in a significant increase in crypt depth. This also suggests that LEP may play a key role in the remodeling of the crypt during the early phases of SBS adaptation. Our observations are consistent with the data of other investigators. Pearson et al. (25) have demonstrated that LEP enhances small intestine carbohydrate absorption beyond the normal adaptive response after massive small bowel resection in a rat. Alavi et al. have shown that systemic LEP administration enhances mucosal mass and absorptive function in normal rat intestine. The authors conclude that LEP seems to be a growth factor for normal small intestine and may play a role in patients who acquire intestinal dysfunction (26).

In this study, we investigated the effects of exogenous LEP on enterocyte turnover (proliferation and apoptosis) in conjunction with long form of the LEPr expression along the villus-crypt axis. Several experimental studies have demonstrated that crypt cell apoptosis progresses quite differently compared with apoptosis along the villi. It should be emphasized that many trophic factors act differentially along the crypt-villus axis in accordance with differential expressions of their receptor $(13,14)$. In a recent study, we have shown that the TGF- $\alpha$ inhibits cell apoptosis in crypts, but it enhances cell apoptosis in villus tips and correlates with different epidermalgrowth factor receptor expressions along the villus-crypt axis (15). In the current experiment, we have shown that the proliferative and anti-apoptotic effect of LEP on enterocyte turnover is strongly correlated with long form of the LEPr expression along the villus-crypt axis. In the crypt compartment, expression of the long form of LEPr increased significantly after bowel resection compared with control animals. Although LEP stimulates proliferation and inhibits apoptosis of epithelial cells, this increase in LEPr expression in crypt cells coincided with increased cell proliferation and decreased cell apoptosis after LEP administration. Both increased cell production and decreased cell death may help to augment SBS adaptation, although future work to confirm this will be needed. This also suggests that LEP may play a key role in the remodeling of the crypt during the early phases of SBS adaptation. In villus tips and lateral villi, cell apoptosis was up-regulated in resected rats compared with Sham rats. Growing evidence suggests that intestinal epithelium, like other rapidly renewing tissues, may process a feedback control whereby cell division in the precursor compartment (the crypts) is regulated by the number of cells in the functional compartment (the villus). It should be emphasized that adaptive stimuli, sufficient to increase significantly the villus cell count, may activate cell death pathways in villus tips and lateral villi. Similar to the crypt compartment, in our experiment long form of the LEPr expression was up-regulated in villus tips in resected rats compared with Sham rats. Because LEP exerts antiapoptotic effects, this increase in LEPr coincides with decreased cell apoptosis in villus tips after LEP administration. Morphologically, this decline in cell apoptosis was reflected in a significant increase in total villus length, suggesting increased surface area. A decrease in enterocyte apoptosis corresponded with the decreased expression of Bax gene expression while $\mathrm{Bcl}-2$ gene expression remained unchanged.

The major flaw of the study is that only the mRNA levels of the long form of the LEPr are analyzed. Further experiments are required to evaluate the expression of the other forms of the LEPr known to exist in various rat tissues.

In conclusion, treatment with LEP stimulates gut growth in rat models of SBS. An increased LEPr mRNA expression along the villus-crypt axis may suggest a relevant role of LEP in the modulation of enterocyte apoptosis within the gastrointestinal mucosa. The effect of LEP on enterocyte turnover is strongly correlated with long form of the LEPr expression along the villus-crypt axis.

\section{REFERENCES}

1. Helmrath MA, Erwin CR, Shin CE, Warner BW 1998 Enterocyte apoptosis is increased following massive small bowel resection. J Gastrointest Surg 2:44-49

2. Thompson JS, Barent B 1999 Effects of intestinal resection on intestinal apoptosis J Gastrointest Surg 3:672-677

3. Stern LE, Falcone RA, Kemp CJ, Stuart LA, Erwin CR, Warner BW 2000 Effect of massive small bowel resection on the $\mathrm{Bax} / \mathrm{Bcl}-\mathrm{w}$ ratio and enterocyte apoptosis. J Gastrointest Surg 4:93-100

4. Falcone RA Jr, Stern LE, Kemp CJ, Shin CE, Erwin CR, Warner BW 1999 Apoptosis and the pattern of DNase I expression following massive small bowel resection. J Surg Res 84:218-222

5. Bjerknes M, Cheng H 1981 The stem cell zone of the small intestinal epithelium. III. Evidence from columnar, enteroendocrine, and mucus cells in the adult mouse. Am J Anat 160:77-91

6. Bjerknes M, Cheng H 1981 The stem cell zone of the small intestinal epithelium. I Evidence from Paneth cells in the adult mouse. Am J Anat 160:51-63

7. Cheng H, Leblond CP 1974 Origin, differentiation and renewal of the four main epithelial cell types in the mouse small intestine. V. Unitarian theory of the origin of the four epithelial cell types. Am J Anat 141:537-561

8. Hall PA, Coates PJ, Ansari B, Hopwood D 1994 Regulation of cell number in the mammalian gastrointestinal tract: the importance of apoptosis. J Cell Sci 107:35693577

9. Kluck RM, Bossy WE, Green DR, Newmeyer DD 1997 The release of cytochrome c from mitochondria: a primary site for Bcl-2 regulation of apoptosis. Science 275:1132-1136

10. Rosse T, Olivier R, Monney L, Rager M, Conus S, Fellay I, Jansen B, Borner C 1998 $\mathrm{Bcl}-2$ prolongs cell survival after Bax-induced release of cytochrome c. Nature 391:496-499

11. Welters CF, Dejong CH, Deutz NE, Heineman E 2002 Intestinal adaptation in short bowel syndrome. ANZ J Surg 72:229-236

12. Westergaard H 2002 Short bowel syndrome. Semin Gastrointest Dis 13:210-220

13. Yang H, Antony PA, Wildhaber BE, Teitelbaym DH 2004 Intestinal intraepithelial lymphocyte gamma delta-T cell-derived keratinocyte growth factor modulates epithelial growth in the mouse. J Immunol 172:4151-4158

14. Warner BW, VanderKolk WE, Can G, Helmrath MA, Shin CF, Erwin CR 1997 Epidermal growth factor receptor expression following small bowel resection. J Surg Res 70:171-177

15. Sukhotnik I, Mogilner JG, Shaoul R, Karry R, Lieber M, Suss-Toby E, Ure BM, Coran AG 2008 Responsiveness of intestinal epithelial cell turnover to TGF- $\alpha$ after bowel resection in a rat is correlated with EGF receptor expression along the villus-crypt axis. Pediatr Surg Int 24:21-28 
16. Bell-Anderson KS, Bryson JM 2004 Leptin as a potential treatment for obesity: progress to date. Treat Endocrinol 3:11-18

17. Badman MK, Flier JS 2005 The gut and energy balance: visceral allies in the obesity wars. Science 307:1909-1914

18. Chaudhary M, Mandir N, FitzGerald AJ, Howard JK, Lord GM, Ghatei MA, Bloom SR, Goodlad RA 2000 Starvation, leptin and epithelial cell proliferation in the gastrointestinal tract of the mouse. Digestion 61:223-229

19. Sukhotnik I, Vadasz Z, Coran AG, Lurie M, Shiloni E, Hatoum OA, Mogilner JG 2006 Effect of leptin on intestinal re-growth following massive small bowel resection in rat. Pediatr Surg Int 22:9-15

20. Chomczynski P 1993 A reagent for the single-step simultaneous isolation of RNA DNA and proteins from cell and tissue samples. Biotechniques 15:532-534:536-537

21. Whang EE, Dunn JC, Joffe H, Mahanty H, Zinner MJ, McFadden DW, Ashley SW 1996 Enterocyte functional adaptation following intestinal resection. J Surg Res 60:370-374
22. Hines OJ, Bilchik AJ, Zinner MJ, Skotzko MJ, Moser AJ, McFadden DW, Ashley SW 1994 Adaptation of the $\mathrm{Na}$ /glucose cotransporter following intestinal resection. J Surg Res 57:22-27

23. Barrenetxe J, Villaro AC, Guembe L, Pascual I, Muñoz-Navas M, Barber A, Lostao MP 2002 Distribution of the long leptin receptor isoform in brush border, basolateral membrane, and cytoplasm of enterocytes. Gut 50:797-802

24. Cammisotto PG, Renaud C, Gingras D, Delvin E, Levy E, Bendayan M 2005 Endocrine and exocrine secretion of leptin by the gastric mucosa. J Histochem Cytochem 53:851-860

25. Pearson PY, O'Connor DM, Schwartz MZ 2001 Novel effect of leptin on smal intestine adaptation. J Surg Res 97:192-195

26. Alavi K, Schwartz MZ, Prasad R, O'connor D, Funanage V 2002 Leptin: a new growth factor for the small intestine. J Pediatr Surg 37:327-330 